\title{
Insightfulness as a dynamic process in development and treatment: a commentary
}

\section{R.M.Pasco Fearon}

\begin{abstract}
This special issue presents a series of conceptually interlinked papers on the construct of insightfulness and its role in child development and intervention. In this commentary, I provide some reflections on the nature of insightfulness from the point of view of developmental and clinical psychology. Four themes are highlighted: 1) the potential role of insightfulness in understanding the parenting mediators of attachment transmission, 2) the role of insightfulness in understanding the connections between early experiences and later social outcomes, 3 ) the dynamic evolution of insightfulness across development and 4) the different elements of insightfulness and their distinct contributions to caregiving behaviour.
\end{abstract}

Keywords: insightfulness, attachment, social competence, sensitivity, intervention

Correspondence:

R.M. Pasco Fearon

Research Department of Clinical, Educational and Health Psychology, University College London

Gower Street

London WC1E 6BT

United Kingdom

Email: p.fearon@ucl.ac.uk 
Learning about others, making sense of them, understanding and relating to them, are profoundly important human skills and activities that surely underpin healthy relationships, be they parent-child relationships, spousal relationships or friendships. Despite the seeming obviousness of this, we are really only just beginning to recognise and understand the role of these processes in scientific terms for child and family development. The current special issue contains a wonderfully rich set of papers all focusing on the construct of insightfulness, which is one of several valuable ways that developmentally-oriented researchers have developed to study this complex and subtle set of processes that we sometimes refer to collectively as mentalizing (Fonagy, Gergely, \& Jurist, 2004). In reading these papers, I was struck by how consistently informative measurements of insightfulness appear to be for learning about parenting and child development. The results of these studies are in line with a recent meta-analysis looking at security and insecurity of attachment (Zeegers, Colonnesi, Stams, \& Meins, 2017), which found attachment to be robustly associated with measures of insightfulness and other related measures of mentalizing, with an average correlation of approximately $r=.30$. This is higher (although not dramatically so) than the meta-analytic average for the association between attachment and observed sensitivity (which is $r \sim .22-.24$, Wolff \& Ijzendoorn, 1997). Many of us have been intrigued by the possibility that insightfulness and other related measures (like mind-mindedness and reflective function) might help us understand the so called 'transmission gap'(Van IJzendoorn, 1995). Of course, none of these measures directly capture parental behaviour, so they cannot fill the gap alone; nevertheless, they may help us look in the right places for parenting mediators of intergenerational transmission. Another issue that struck me as I read the papers was how each of them, in different ways, addressed issues, or raised questions, about how insightfulness has its effects - across time, or across contexts. As a measure of how one thinks about a particular relationship, here and now, how does it relate to earlier experiences and how does it relate to other relationships, now and in the future? And can insightfulness be changed, and if so, how?

The issue of how early infant-parent experiences are carried forward to affect later social relationships is of particular interest to me, and has been highlighted by the accumulating evidence that attachment insecurity is robustly associated with poorer social competence and particularly with the quality of relationships with new or unfamiliar peers (Groh et al., 2014). It is surprising how little we know about the psychological mechanisms connecting these two important developmental processes. The traditional explanation of course rests on the idea that early experiences have their effects on later outcomes through the action of internal working models of attachment, which shape the child's interpretation of, and responses to, important later social experiences. Although social cognitive research has provided some, albeit currently quite limited, evidence in support of this idea (e.g., see Sherman, Rice, \& Cassidy, 2015), it is remarkable how little this topic has been investigated to any depth. The insightfulness or mentalizing framework provides a novel and complementary way of thinking about these interconnections over development. Clearly, being able to think about other people in a compassionate way, as three-dimensional psychological agents, with complex feelings and motivations that are not the same as one's own, is a critical dimension of personality functioning and no doubt plays a vital role in children's social relationships. This is probably particularly important as children grow up and their relationships become more complex and more intimate. To the extent that these capacities have their roots in early attachment experiences, they may provide a powerful way of understanding how and why early experiences affect later social functioning. 
The paper by Shahar-Maharik is therefore particularly interesting. They showed that children whose early experiences were characterised by a parent who was able to think insightfully about their experiences and who provided a secure base for their attachment needs were more likely to be able to think in similarly sophisticated, well-rounded ways in their own relationships with peers in adolescence. So, long-term connections between early supportive care, or secure attachment, may indeed be mediated by continuities in the child's capacity to mentalise others.

As always one is still left wondering about mechanisms: for example, how is it that parental insightfulness influences the development of the child's later insightfulness? Is this, as Fonagy and colleagues would argue (Fonagy et al., 2004), a result of early marked mirroring and affect labelling, which promotes the child's own self-understanding? Or should we understand this as a kind of social modelling, in which the parent demonstrates how "we" as a family treat others, think about others and look after others? What other learning mechanisms might be involved? We might also wonder to what extent these connections between earlier and later outcomes are rooted in stable psychological structures engendered in the child early on or are reflections of the ongoing way in which the family inter-relates. These are important matters because they relate to the extent to which change might be possible and also to how it might be done.

In our work on Mentalization-Based Treatment for Families (MBT-F, Keaveny, Midgely, Asen, Bevington et al., 2012), we take the view that a great deal of the low insightfulness that can characterise struggling families does not reflect a lack of basic capacity for insight. Instead, we think of this as resulting from the effects of chronic stress and chronic patterns of unsupportive and non-mentalizing family interactions. These chronic patterns of interaction make seeing or understanding each other in new ways very difficult. The challenge often seems to be to find some way of creating a sense of curiosity in the minds of family members and, a sense of sufficient security in the moment, to explore. If that can be achieved it is remarkable how much insightfulness can be restored and how non-mentalizing can gradually be unravelled, as each member of the family feels heard and understood by other members of the family. This suggests to me that a lot of non-mentalizing is maintained by ongoing, hereand-now, patterns of interaction which have become highly stable over time.

That is not to say of course that all families and all individuals are equally prone to nonmentalizing, or that early experiences do not play a crucial role in biasing the trajectory that these family interactions take. No doubt there is a complicated interaction between the past and the here-and-now that takes place. In contemporary developmental psychology, we tend not consider constructs like defences, but our experience (not unique to us of course) is that a great deal of non-mentalizing arises as a kind of defence against perceived threats to the self. Defensively interpreting another person's actions in one rigid way of course tends to lead one to act rigidly and aggressively, which creates a sense of threat, defensiveness and nonmentalizing in others, and to behave in similarly coercive ways in return. This is a key way of thinking about how mentalizing impacts on family systems in our mentalization based treatment work. It also is also a helpful way of thinking about how mentalizing and insightfulness might relate to social behaviour in close relationships in general in other social groups. Peers perhaps represent one of the most important of these, particularly in adolescence.

Another interesting issue raised by this paper is whether insightfulness might be just as important if not more important in the evolution of new relationships (i.e. with initially 
unfamiliar peers) than with established close friendships (the latter being the focus of the Shahar-Maharik paper). In our meta-analysis (Groh et al., 2014) we found that attachment played a larger role in more unfamiliar, less close, relationships than in relationships with close friends. Related to that, a fascinating topic that could be considered in future research is how relationships with peers evolve over time and what role insightfulness plays in that, both as the relationship is beginning and subsequently. There are obvious life transitions where these relationship formation processes can be studied very naturally, for example at the beginning of secondary school, at the start of a foster care placement, or indeed at the start of college or university. What role does insightfulness play in navigating the challenges and uncertainties inherent to the beginning of a relationship? What role does it play in repairing problems that inevitably occur, which might be make-or-break for that relationship in its early phase, in contrast to more established relationships? The possibility that insightfulness itself evolves during the course of a relationship is very much highlighted by the finding reported in the Shahar-Maharik paper that more long-standing relationships tended to show greater insight than briefer ones. This is an intriguing finding: as the authors note, it could of course represent an association between insight and how long a relationship lasts, rather than an effect of time on insight. But the possibility that insightfulness might be dynamically changing as the relationship develops is intriguing. A longitudinal study is really needed to look at this properly.

This also highlights another fascinating question: to what extent is a relationship determined by the respective insightfulness of each party? In typical research designs we tend to look at only one member of a peer relationship (the 'target' child who is part of our cohort), so the role of the peer's insightfulness gets obscured. Furthermore, as the relationship develops does each party's insightfulness regarding that relationship change in similar ways or towards a similar outcome, or are they largely shaped by their respective (potentially quite different) attachment histories?

It was very nice to read the authors concluding remarks, where they spoke so positively about the ability of adolescents to be insightful, and to think compassionately and coherently about others, when stereotypes of adolescence would have us think quite differently about them. I couldn't agree more with the authors about this and never cease to be impressed by how articulate and thoughtful young people can be, particularly when they are treated as people capable of being so. In MBT-F work, we often notice how dependent the insightfulness of a young person is on how comfortable they feel in a social situation. For example, stony silence or rigid dismissiveness might be all that we see during a difficult conversation in the presence of parents, but this can be dramatically contradicted by their thoughtfulness and insight when away from the heat of the family interaction, when they are supported by the therapist and made to feel confident they will be listened to. Again, this highlights the dynamic, and possibly context dependent, nature of insightfulness or mentalization.

The paper by Gomez and colleagues begins by making a intriguing connection between two crucial facets of sensitive parenting: support for the child's need for proximity and support for the child's need for autonomy and exploration, and two facets of insightfulness: the parents understanding and acceptance of the child's feelings and wishes versus the parent's capacity to see the child as separate from them. This way of thinking of course draws us closer to ways of understanding the specific cognitive processes that motivate different dimensions of parental behaviour. This is a crucial missing part of the puzzle within attachment research and in parenting research more generally. The tendency within most previous research has been to capture global stylistic differences in the way a parent thinks and relate this to global 
stylistic differences in the way they behave with the child. This has been valuable, but only takes us so far in terms of understanding precisely what the underlying cognitive and behavioural mechanisms are, which of course is crucial when thinking about intervention. In drawing attention to the difference between support for autonomy or scaffolding (i.e. nonintrusiveness) and support for contact or comfort, the authors highlight the importance of disentangling different parenting features and determining which are most closely and causally involved in which different outcomes. This unpacking process has been growing within the developmental psychology literature recently (e.g., Leerkes et al., 2015) and is an important innovation. To take the authors' line of thinking a little further, one would imagine that support for cognitive development would involve both understanding that the child needs to discover solutions to problems or learn by trial and error and an ability to notice and correctly identify when the child needs a 'leg up', to progress their learning. In that sense, non-intrusiveness (the most robust predictor of cognitive outcome in the Gomez study) alone must not be sufficient - there must surely be a continuous ebb and flow of responsiveness/support and autonomy promotion, led by a moment-to-moment understanding of what the child needs right now. This example also illustrates how precise and dynamic different parental cognitions and behaviours are likely to be in relation to unfolding child behaviour and how large the gap tends to be between how we measure such processes and how we think they probably work.

In the paper by Martinez, insightfulness becomes a construct that can also be thought about as a personal protective factor. These authors found in their longitudinal study that high levels of maternal insightfulness acted as a kind of buffer, helping, it seems, mothers to maintain consistent levels of sensitive and responsive caregiving in the face of stressful life experiences. Put the other way around, less insightful mothers appear to be more prone to becoming negative in their interactions with their infant in the face of life stresses. To link this back to the earlier discussion, the study presents an interesting question about whether the insightfulness that is being studied here reflects the mother's understanding and capacity to empathise with this particular child which then buffers this particular relationship from external stressors or whether insightfulness more generally (i.e. not specifically with respect to this mother-infant dyad) is reflective of a personal resilience factor that is broader in nature (e.g., related to free-autonomous states of mind with respect to attachment). Relatedly, insightfulness may be associated with greater self-insight, which in turn may help adults manage their emotions and use their social support networks more effectively in times of stress, which may then be protective of their mother-child relationship. Another possibility is that the apparent resilience does not arise from insightfulness as such but from some other third variable. And of course all of these possibilities may be partially true. Understanding and disentangling these different processes would be very valuable targets of future research. Intervention studies could be particularly interesting in that regard because interventions could be focused on improving insightfulness within a dyad or improving insightfulness as a "generic" personal ability (e.g., psychotherapeutically) and tracking the putative mechanistic pathways that might be involved.

All of these issues regarding the relational specificity of insightfulness, and the capacity for difference and change, are neatly distilled in the paper by Siller et al. They begin their overview by taking the explicit position that the capacity for reflection and thoughtful insight into a child's mind is a specific process occurring in the context of that relationship and its history of interactions, rather than a domain general trait, state of mind or capacity of the parent. As noted earlier, the degree of specificity and of plasticity is extremely important for thinking about modes of intervention. In the Siller et al paper the assumption is made that 
mindfulness is open to change through an intervention that focuses on immediate interactions. The results of the randomised trial provide some direct, albeit preliminary, support for the idea that insightfulness can be improved through an interaction-focused intervention, which is good news from the point of view of supporting families and children and very interesting theoretically, because it suggests that insightfulness is dynamic and tied to interactions within a specific relationship. A key question is whether the changes in insightfulness Siller et al observed generalise to other domains; for example do they generalise to improved parenting and improved dyadic interactions? We know already that insightfulness is correlated with responsive caregiving cross-sectionally (as the paper by $\mathrm{Ziv}$ and colleagues shows in this issue), but it is not well established whether changes in insightfulness lead to changes in responsive care. There is good reason to be optimistic that it will, and I look forward to seeing more intervention work testing out this possibility. Another interesting issue that future research could explore is whether changes in insightfulness generalise beyond the specific relationship. Most parents who take part in interventions of this kind will naturally think about how the discoveries they are making during the clinical work apply to other children they care for or indeed to other non-parental relationships, so there is certainly potential for generalisation. An immediate question for this study is whether the apparent change in mindfulness was a real change - the intervention involved video feedback so it is conceivable that there was some element of 'teaching to the test' because the insightfulness assessment involves quite a similar process. That is important to establish, given that this study shows such promising results.

One of the apparent paradoxes in this area is that we know already that early life experiences can affect insightfulness and indeed the work reported in this issue by $\mathrm{Ziv}$ and colleagues provides further evidence of this. How is it that these apparently long-standing vulnerabilities lead to difficulties with insightfulness that may also be, at least to a degree, relationshipspecific? It seems that insightfulness within a specific relationship may be the result of a continuous interaction between one's personal vulnerabilities and the opportunities and challenges one encounters when interacting within a specific relationship. That is an important thing to keep in mind, and highlights three issues: a) it may be valuable to target interventions to those with such personal vulnerabilities (as suggested by Siller and colleagues), b) that improvements in insightfulness may occur even when earlier traumas and other vulnerabilities have not been directly addressed or resolved, and c) improvements in mindfulness within a relationship may nevertheless retain a degree of vulnerability, for example in other relationships or when the current relationship presents new challenges. With the latter point in mind, in the future it may be worth considering the inclusion of so-called booster sessions to help the relationship continue on a positive trajectory.

The special issue is rich food for thought and shows clearly that the construct of insightfulness has a great deal to offer both developmental researchers and clinicians.

\section{References}

Fonagy, P., Gergely, G., \& Jurist, E. L. (2004). Affect regulation, mentalization and the development of the self: Karnac books.

Groh, A. M., Fearon, R. P., Bakermans-Kranenburg, M. J., Van IJzendoorn, M. H., Steele, R. D., \& Roisman, G. I. (2014). The significance of attachment security for children's social competence with peers: A meta-analytic study. Attachment \& Human Development, 16(2), 103-136. 
Keaveny, E., Midgley, N., Asen, E., Bevington, D., Fearon, P., Fonagy, P., Jennings-Hobbs, R. \& Wood, S. (2012) Minding the family mind: the development and initial evaluation of mentalization-based treatment for families. In N. Midgley \& I. Vrouva (Eds), Minding the Child: Mentalization-Based Interventions with Children, Young People and their Families. Taylor \& Francis, London, pp. 98-112.

Leerkes, E. M., Supple, A. J., O'Brien, M., Calkins, S. D., Haltigan, J. D., Wong, M. S., \& Fortuna, K. (2015). Antecedents of maternal sensitivity during distressing tasks: Integrating attachment, social information processing, and psychobiological perspectives. Child Development, 86(1), 94-111.

Sherman, L. J., Rice, K., \& Cassidy, J. (2015). Infant capacities related to building internal working models of attachment figures: A theoretical and empirical review. Developmental Review, 37, 109-141.

Van IJzendoorn, M. H. (1995). Adult attachment representations, parental responsiveness, and infant attachment: a meta-analysis on the predictive validity of the Adult Attachment Interview. Psychological Bulletin, 117(3), 387.

Wolff, M. S., \& Ijzendoorn, M. H. (1997). Sensitivity and attachment: A meta-analysis on parental antecedents of infant attachment. Child Development, 68(4), 571-591.

Zeegers, M. A., Colonnesi, C., Stams, G.-J. J., \& Meins, E. (2017). Mind matters: A metaanalysis on parental mentalization and sensitivity as predictors of infant-parent attachment. Psychological Bulletin, 143(12), 1245. 